\title{
Analysis of Polymorphs Using Simultaneous X-ray Fluorescence and Diffraction with an Imaging Spectrometer
}

\author{
Jeffrey M. Davis ${ }^{1, *}$, Julia Schmidt ${ }^{1}$, Martin Huth ${ }^{1}$, Robert Hartmann ${ }^{2}$, Sebastian Ihle ${ }^{1}$, Daniel Steigenhöfer ${ }^{1}$, \\ Heike Soltau ${ }^{1}$, Lothar Strüder ${ }^{2}$ \\ 1. PNDetector GmbH, Otto-Hahn-Ring 6, 81739 München, Germany \\ 2. PNSensor GmbH, Otto-Hahn-Ring 6, 81739 München, Germany
}

X-ray fluorescence (XRF) is a widely used method for materials characterization. However, for polymorphs such as Rutile and Anatase, both of which are $\mathrm{TiO}_{2}$, the fluorescence spectra will be nearly identical. Differentiating between these polymorphs requires a second measurement with an X-ray diffractometer. Using two instruments increases the cost of the measurement, and it introduces issues with image registration and sample preparation. Previously, simultaneous XRD-XRF has been done by attaching a silicon drift detector (SDD) to an X-ray diffraction (XRD) system. However, due to the typical geometry and source monochromation of an XRD system, the count rate in the SDD is typically low, making the measurement process slow [1]. Overcoming these limitations requires a detector that is both position sensitive and energy dispersive.

The system proposed here uses the pnCCD as an imaging spectrometer to detect both the fluorescence and diffraction signals [2]. As an imaging spectrometer, the pnCCD records both the position and energy of each X-ray detected. It has energy resolution of $150 \mathrm{eV}$ full width at half maximum for $\mathrm{Mn} \mathrm{K} \alpha$, and it has a pixel size of $48 \mu \mathrm{m}$ with subpixel resolution down to $10 \mu \mathrm{m}$. This combined, single instrument measurement technique is possible because fluorescence signals show almost no angular dependence, while diffraction signals do. Diffracted X-rays strictly obey Bragg's law, and the angle of the diffraction can be calculated from the wavelength of the X-ray and the d-spacing of the crystal lattice. A detector that records the position and energy of each X-ray event is necessary to make this distinction, making the pnCCD an excellent detector for these analyses [3].

Figure 1 shows the setup of the experiment, consisting of a simple $30 \mathrm{~W}$ Mo X-ray tube and the pnCCD camera in the forward scattering (Bragg-Brentano) geometry. Because the X-ray source is polychromatic, a wide range of d-spacings can be investigated, and a diffraction pattern can be derived without moving the pnCCD. Measuring the distance from the sample to the center of the pnCCD provides a simple method to calculate the $2 \theta$ value of each pixel in the detector. The $2 \theta$ range of the measurement is significantly larger than the geometric range due to the polychromatic X-rays from the source and the energy dispersive capability of the detector. Determining the $2 \theta$ angle and measuring the energy of the incoming X-ray, the corresponding d-spacing of each pixel on the pnCCD could be calculated from Bragg's law. Through Bragg's law, the d-spacings can then be converted into a diffraction pattern similar to those in the International Center for Diffraction Data (ICDD) database. Here, samples of the $\mathrm{TiO}_{2}$ polymorphs Rutile and Anatase were analyzed in the XRD-XRF system. The X-ray data were saved in the X-ray spectrum image format (a complete X-ray spectrum at each pixel). Processing each X-ray image in the cube according to the angular dependence process described above produces the spectra shown in Figure 2. Although the fluorescence spectra for the polymorphs are nearly identical, the difference in the diffraction patterns is obvious. The pnCCD makes it possible to differentiate these polymorphs with a single measurement. 


\section{References:}

[1] Luggar, R. D., Farquharson, M. J., Horrocks, J. A., et al, Multivariate analysis of statistically poor EDXRD spectra for the detection of concealed explosives, X-ray Spectrometry 27 (1998) p. 87-94

[2] Scharf, O., Ihle, S., Ordavo, I., et al, Compact pnCCD-based X-ray camera with high spatial and energy resolution: A color X-ray camera, Analytical Chemistry, 83 (2011), p. 2532:2538.

[3] Leitenberger, W., Hartmann, R., Pietsch, U., et al, Application of a pnCCD in X-ray diffraction: a three-dimensional X-ray detector, Journal of Synchrotron Radiation, 15 (2008), p.449:457.

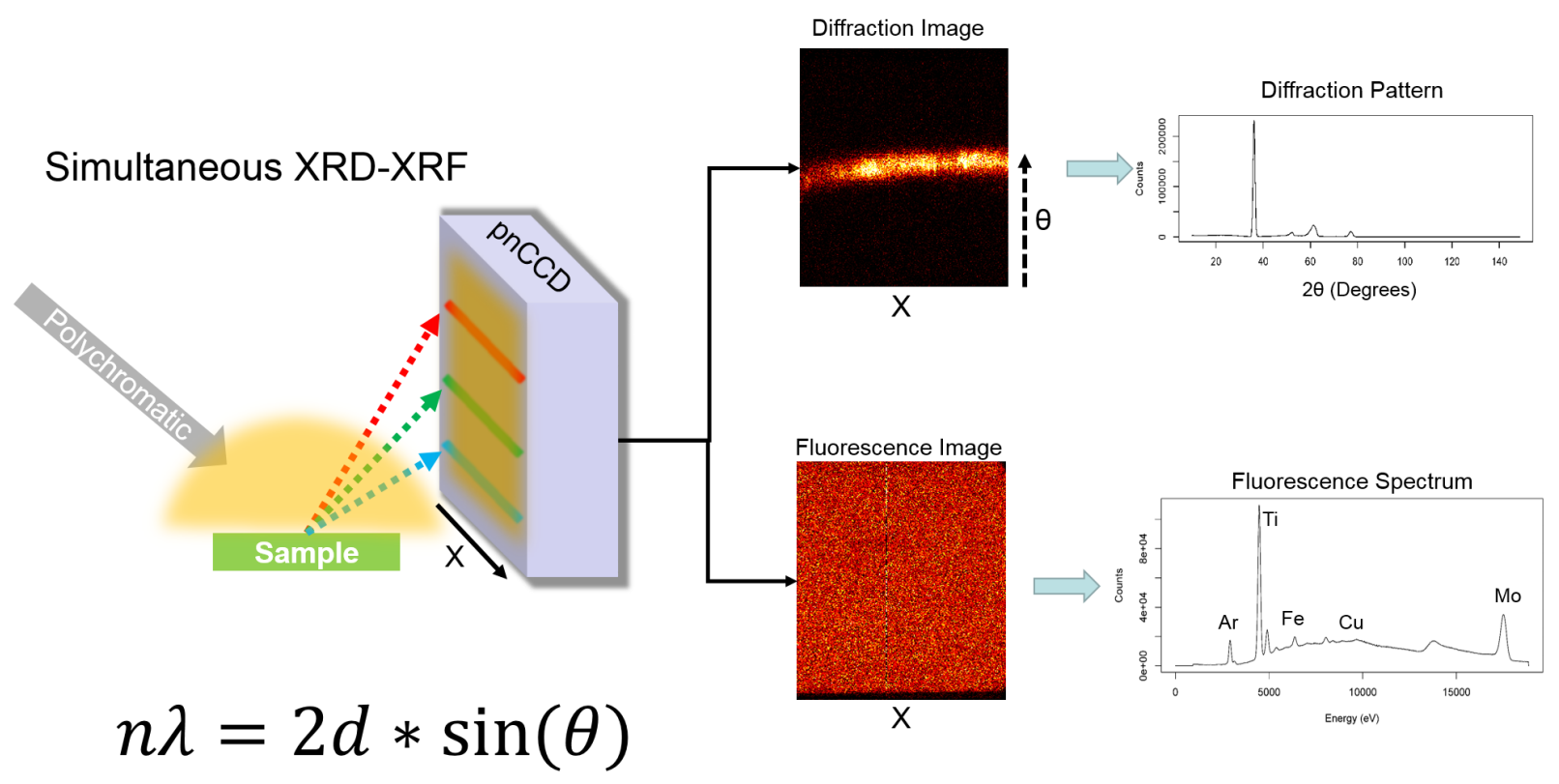

Figure 1: A diagram showing the differentiation of XRD and XRF signals in the pnCCD. Diffracted $\mathrm{X}$-rays have energy and angular dependence, appearing as lines in an X-ray image, while fluoresced X-rays have no angular dependence. Using image processing of the data, these signals can be separated out and turned into diffraction and fluorescence spectra.
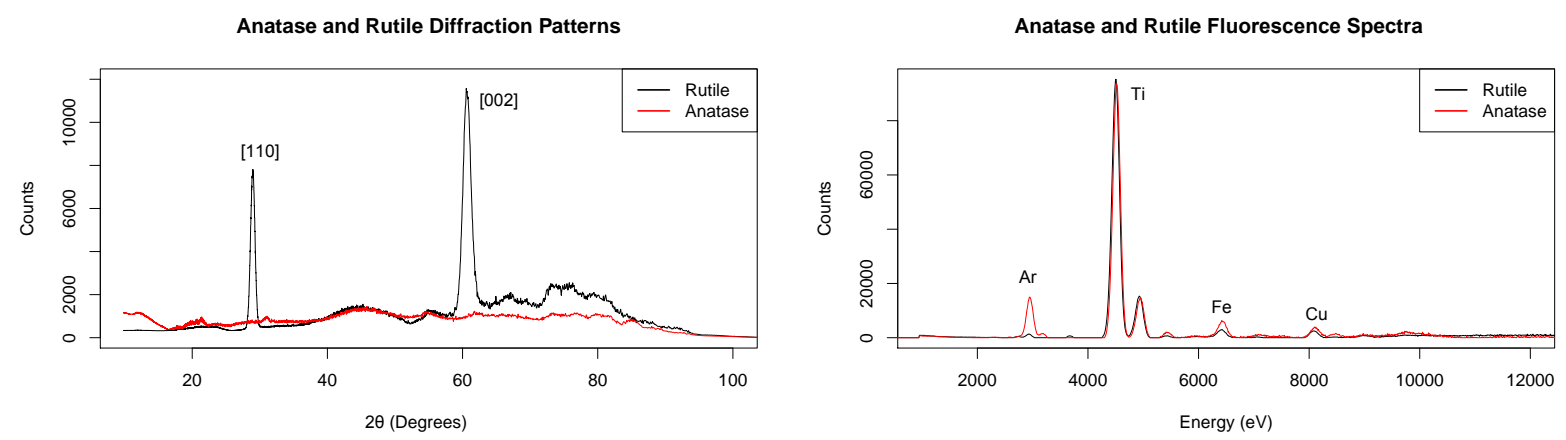

Figure 2: Corresponding diffraction patterns (left) and fluorescence spectra (right) for samples of Anatase and Rutile, which are compositionally identical but differ in crystal structure. 